\title{
Experimental and computational investigation of graphene/SAMs/n-Si Schottky diodes
}

\author{
H. Aydin ${ }^{\mathrm{a}, *}$, C. Bacaksiz ${ }^{\mathrm{b}}$, N. Yagmurcukardes ${ }^{\mathrm{a}}$, C. Karakaya ${ }^{\mathrm{c}, \mathrm{d}}$, O. Mermer ${ }^{\mathrm{e}}$, M. Can ${ }^{\mathrm{f}}$, \\ R.T. Senger ${ }^{\text {b, h }}$, H. Sahin ${ }^{g, h}$, Y. Selamet ${ }^{b}$ \\ a Department of Material Science and Engineering, Izmir Institute of Technology, 35430, Izmir, Turkey \\ b Department of Physics, Izmir Institute of Technology, 35430, Izmir, Turkey \\ c Department of Metallurgy and Materials Engineering, Manisa Celal Bayar University, 45140, Izmir, Turkey \\ d Department of Material Science and Engineering, Izmir Katip Celebi University, 35620, Izmir, Turkey \\ e Department of Electrical and Electronics Engineering, Ege University, 35100 Izmir, Turkey \\ ${ }^{\mathrm{f}}$ Department of Engineering Sciences, Izmir Katip Celebi University, 35620 Izmir, Turkey \\ ${ }^{g}$ Department of Photonics, Izmir Institute of Technology, 35430 Izmir, Turkey \\ h ICTP-ECAR Eurasian Center for Advanced Research, Izmir Institute of Technology, 35430 Izmir, Turkey
}

\section{A R T I C L E I N F O}

\section{Article history:}

Received 7 March 2017

Received in revised form 19 July 2017

Accepted 24 September 2017

Available online 29 September 2017

\section{Keywords:}

Graphene

Self-assembled monolayers (SAMs)

Schottky diode

\begin{abstract}
A B S T R A C T
We have investigated the effect of two different self-assembled monolayers (SAMs) on electrical characteristics of bilayer graphene (BLG)/n-Si Schottky diodes. Novel $4^{\prime \prime}$ bis(diphenylamino)-1, $1^{\prime}: 3^{\prime \prime}-$ terphenyl-5' carboxylic acids (TPA) and 4,4-di-9H-carbazol-9-yl-1,1': $3^{\prime} 1^{\prime}$-terphenyl-5' carboxylic acid (CAR) aromatic SAMs have been used to modify n-Si surfaces. Cyclic voltammetry (CV) and Kelvin probe force microscopy (KPFM) results have been evaluated to verify the modification of $n$-Si surface. The current-voltage $(I-V)$ characteristics of bare and SAMs modified devices show rectification behaviour verifying a Schottky junction at the interface. The ideality factors $(n)$ from $\ln (I)-V$ dependences were determined as 2.13, 1.96 and 2.07 for BLG/n-Si, BLG/TPA/n-Si and BLG/CAR/n-Si Schottky diodes, respectively. In addition, Schottky barrier height $(\mathrm{SBH})$ and series resistance $\left(R_{S}\right)$ of SAMs modified diodes were decreased compared to bare diode due to the formation of a compatible interface between graphene and Si as well as $\pi-\pi$ interaction between aromatic SAMs and graphene. The CAR-based device exhibits better diode characteristic compared to the TPA-based device. Computational simulations show that the BLG/CAR system exhibits smaller energy-level-differences than the BLG/TPA, which supports the experimental findings of a lower Schottky barrier and series resistance in BLG/CAR diode.
\end{abstract}

(C) 2017 Elsevier B.V. All rights reserved.

\section{Introduction}

Graphene is a one atom thick sheet of $s p^{2}$ bonded carbon atoms arranged in a honeycomb crystal lattice that can be thought of as two dimensional material [1]. Linear energy-wave vector relation [1,2], zero band gap and high charge carrier mobility [3] of graphene make it promising material for optoelectronic applications.

Understanding of metal-semiconductor Schottky junction is essential for the improvement of technological device applications. In addition, owing to the unique properties and easy thin film processing, graphene can be easily combined with semiconducting materials and formed graphene-semiconductor heterojunction. The graphene-semiconductor based Schottky junction is one of the devices which is used in many applications including barristors [4],

\footnotetext{
* Corresponding author.

E-mail address: hasanaydin@iyte.edu.tr (H. Aydin).
}

solar cells [5], photodetectors [6] and sensors [7] and have been studied on various semiconductor materials such as GaAs [8], SiC [9], GaN [10] and Si [11]. Among these materials, Si is more convenient material as it is the non-toxic, rich in the nature, mostly prefferred material in integrated electronics and has large built-in field with graphene $\left(V_{b i}=0.55-0.75 \mathrm{~V}\right)$ [11].

Structural differences between the graphene and Si lead to the formation of defects at the interface and limits the stability and performance of the Schottky devices. Recent studies have shown that, the graphene-Si Schottky junctions have focused on effect of different annealing temperatures on electronic transport behaviour [12-15] and determination of Schottky barrier height, ideality factor and series resistance parameters from currentvoltage characteristic [16-18]. However, there is still lack of studies on improving charge transfer between graphene-Si interface.

SAMs are a good choice for the modifying Si surface to provide compatible interface between graphene and $\mathrm{Si}$, as well as they enhance charge transfer from Si surface to graphene. Addi- 


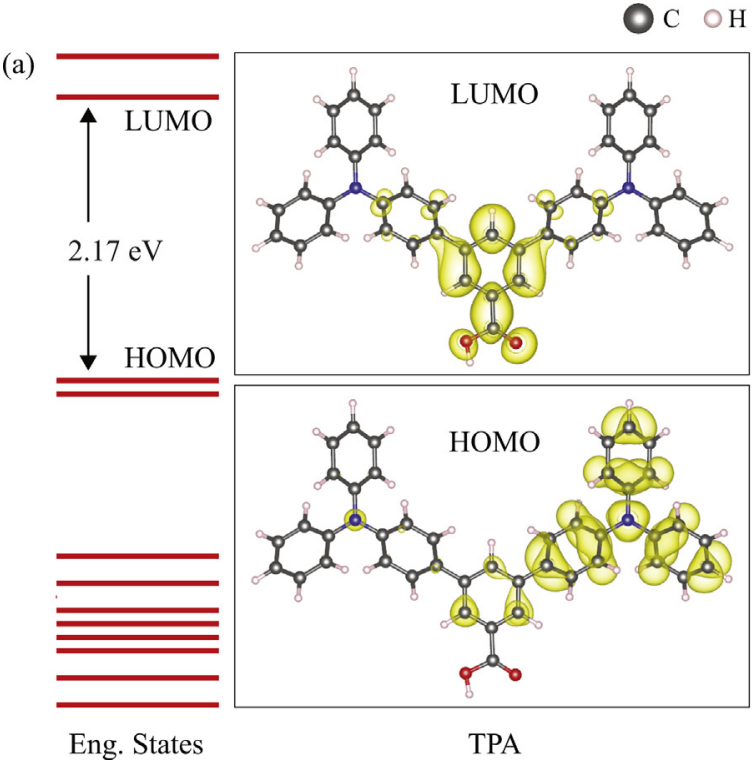

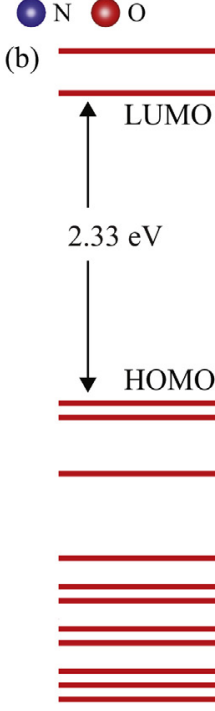

Eng. States

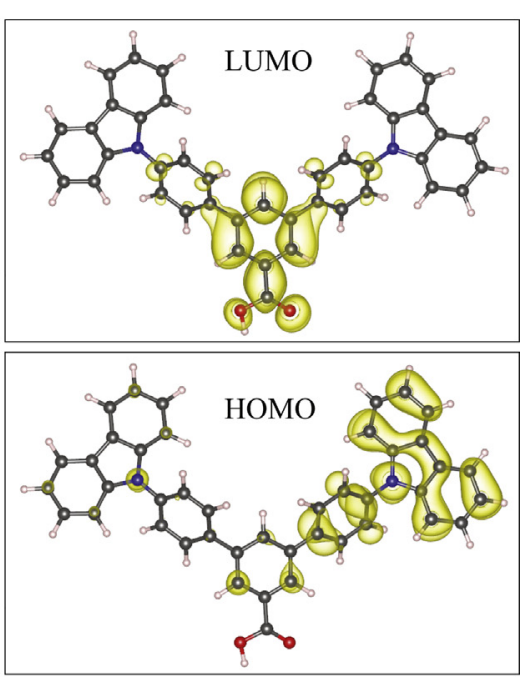

CAR

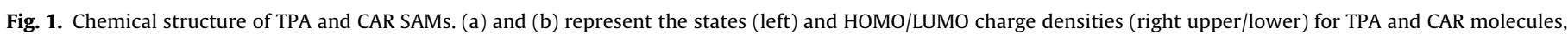
respectively.
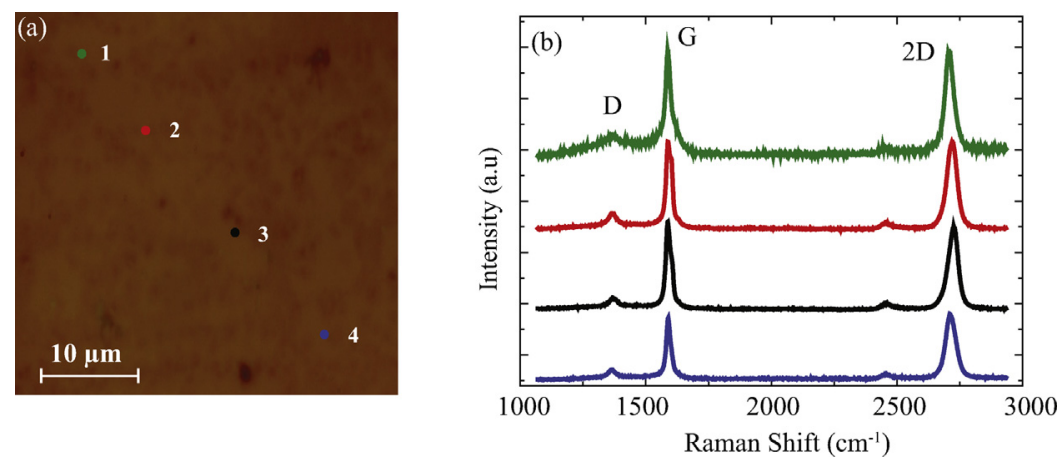

Fig. 2. Multi points Raman spectra of bilayer graphene transferred on $\mathrm{SiO}_{2} / \mathrm{Si}$.

tionally, SAMs are promising materials in organic electronics due to the their flexibility in the chemical structure (choosing convenient donor and acceptor groups) [19], ability in changing the physical properties (such as absorbance spectrum, extinction coefficient and energy levels) [20], easy to synthesized and purified $[19,20]$. In recent studies, SAMs in graphene field effect transistors have been used as a seed layer in graphene-dielectric interface to improve device performance $[21,22,23,24-30,35]$. Common aim of these studies are to passivate dielectric surface with SAMs resulting in reducing charge traps at the graphene-dielectric interface, limiting unintentional doping and increasing field effect mobility. Moreover, the theoretical calculation assisted experimental studies on SAM systems were previously reported [31-33]. However, to our knowledge, the SAMs based graphene Schottky junction has not been reported so far, except our previous work [34].

In this study, TPA and CAR aromatic SAMs were used to modify $n$-Si surface. In order to confirm the modification of SAMs, CV and KPFM measurements were performed on bare and SAMs modified n-Si surfaces. In addition, Raman Spectroscopy was used to define quality and number of graphene layers. Furthermore, electrical characteristics of graphene based Schottky junctions were investigated after the modification of $n$-Si substrates with TPA and CAR SAMs. The results show that SAMs modified devices exhibit better diode performance than bare device in terms of turn-on voltage, barrier height and series resistance. The DFT based electronic property and work function calculations of Si surface, single TPA and CAR molecules, and graphene also confirm the experimental results.

\section{Experimental and computational methodology}

\subsection{Preparation of SAMs}

Commercial $\mathrm{SiO}_{2}(300 \mathrm{~nm}) / \mathrm{n}$-Si (University Wafer) with resistivity of $\rho=1-10 \Omega \mathrm{cm}$ substrates were cleaned in ultrasonic bath for $10 \mathrm{~min}$ in deionized water, acetone, ethanol and 2-proponal, respectively. TPA and CAR aromatic small molecules with carboxylic acid were used as SAMs. SAMs with $1 \mathrm{mM}$ concentration were prepared at room temperature in dimethyl sulfoxide (DMSO). $\mathrm{SiO}_{2}(300 \mathrm{~nm}) / \mathrm{n}$-Si substrates were kept in DMSO-SAMs solution for $24 \mathrm{~h}$ to be covered with TPA and CAR SAMs. The substrates were then rinsed with DMSO to remove SAMs residues and dried in a stream of $\mathrm{N}_{2}$ gas.

\subsection{Graphene growth and transfer process}

$\mathrm{Cu}$ foil $(25 \mu \mathrm{m}$ thick, 99.8 purity, Alfa Aesar) as catalystsubstrate material, was placed on a quartz glass in the atmospheric pressure chemical vapor deposition (CVD) system. First of all, the $\mathrm{Cu}$ foil was heated up to $1073^{\circ} \mathrm{C}$ under $\mathrm{H}_{2}(20 \mathrm{sccm})$ and $\operatorname{Ar}(200 \mathrm{sccm})$ with a ramp of $30^{\circ} \mathrm{C} \mathrm{min}^{-1}$ then annealed under same temperature and flow rates for $1 \mathrm{~h}$. After annealing process, $\mathrm{CH}_{4}(10 \mathrm{sccm})$ was 

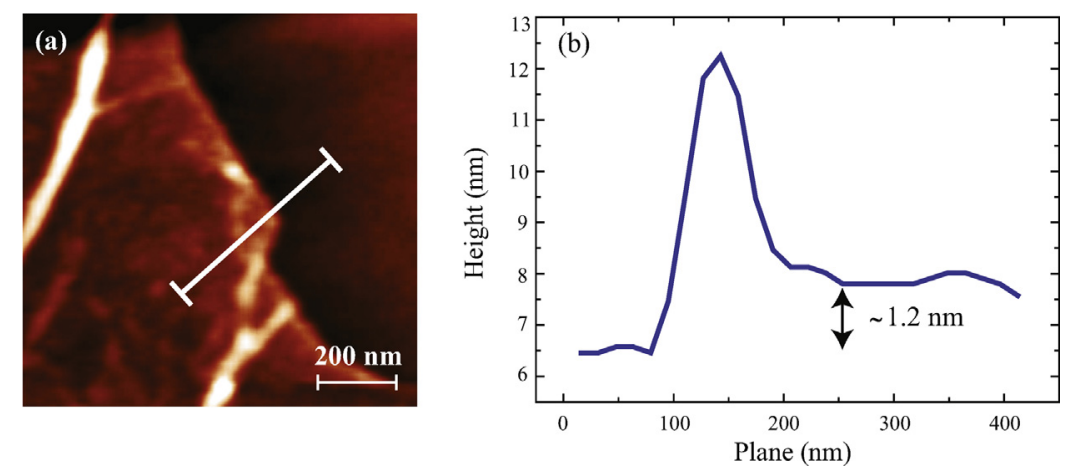

Fig. 3. (a) AFM image of graphene film transferred on $\mathrm{SiO}_{2} / \mathrm{Si}$ and (b) cross-section analysis of graphene films.
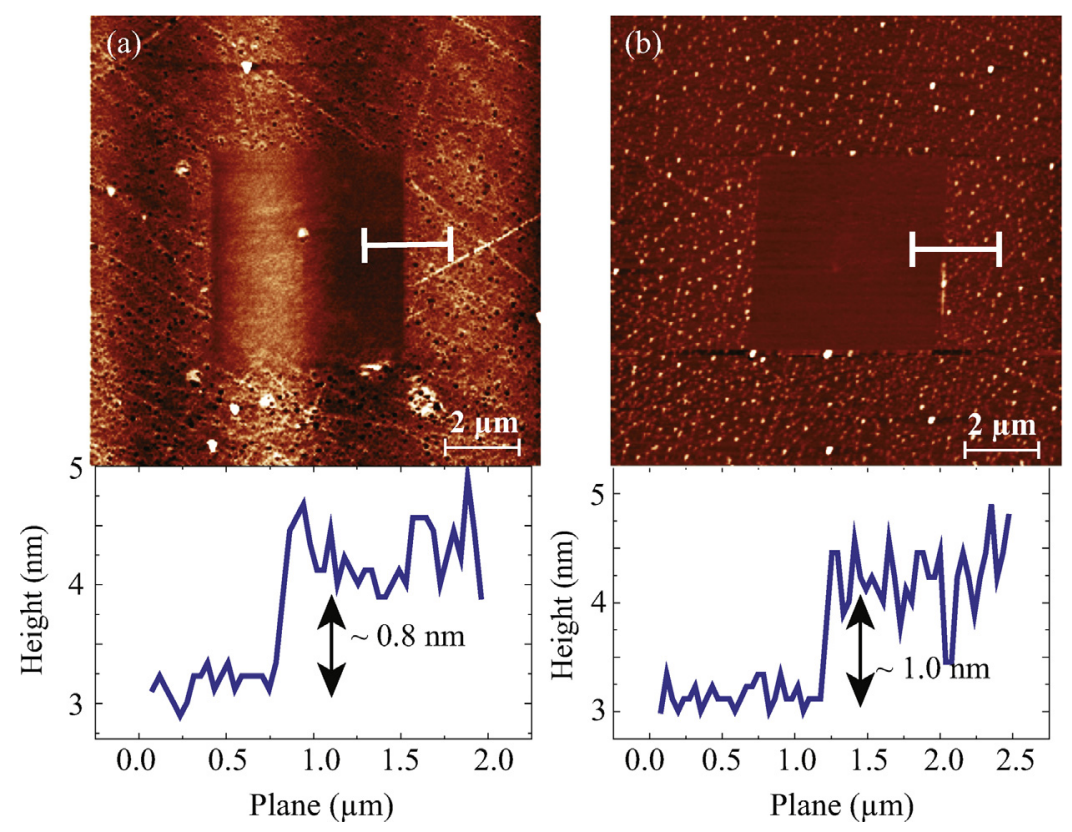

Fig. 4. AFM morphology and height profile of scratch lithography in contact mode operation, (a) TPA and (b) CAR SAMs on n-Si surface.

introduced into tube furnace for three minutes in order to obtain graphene growth. Finally, the sample was left for rapid cooling down to the room temperature in a gas flows of $\mathrm{H}_{2}(20 \mathrm{sccm})$ and Ar $(200 \mathrm{sccm})$. Microposit S1318 Photoresist (PR) was utilized as supporting layer during the graphene transfer process. We drop casted on the graphene- $\mathrm{Cu}$ substrate and annealed overnight at $70{ }^{\circ} \mathrm{C}$. Iron chloride $\left(\mathrm{FeCl}_{3}\right)$ solution was used for $\mathrm{Cu}$ etching. As the $\mathrm{Cu}$ foil fully etched away, graphene-PR was rinsed by deionized water to remove $\mathrm{FeCl}_{3}$ residue. After $\mathrm{N}_{2}$ drying, graphene-PR was transferred onto clean $\mathrm{SAMs} / \mathrm{SiO}_{2} / \mathrm{n}$-Si substrate and bare $\mathrm{SiO}_{2} / \mathrm{n}$ Si substrate. Thereafter, substrates were baked at $110^{\circ} \mathrm{C}$ in order to provide better adhesion of graphene to the substrates. Finally, PR was removed in hot acetone to yield large area graphene on $\mathrm{SAMs} / \mathrm{SiO}_{2} / \mathrm{n}-\mathrm{Si}$ and $\mathrm{SiO}_{2} / \mathrm{n}-\mathrm{Si}$ substrate. We believe that during the graphene transfer process, acetone does not dissolve the SAMs molecules on the n-Si due to occurred chemical bonding between SAMs molecules and n-Si. Additionally, there is no degradation in any SAMs molecules because solvent of SAMs molecules is DMSO. Moreover, annealing of $n-S i$ samples during the graphene transfer improves the SAM surface morphologies including increment of the ordered domains and reduction of the number of vacancy islands [36].

\subsection{Device fabrication and characterization}

We etched a part of $\mathrm{SiO}_{2}$ using mixture of $\mathrm{H}_{2} \mathrm{O}: \mathrm{HNO}_{3}: \mathrm{HF}$ (60:1:1.5) to prevent electrical shortening along the graphene layer. Then, graphene transferred placed on etched $\mathrm{SiO}_{2} / n-\mathrm{Si}$ substrate with regarding the surface colour changes between $\mathrm{SiO}_{2}$ and n-Si. While chromium ( $\mathrm{Cr}$ ) and gold ( $\mathrm{Au}$ ) electrodes with a thickness of 10 and $100 \mathrm{~nm}$ were deposited on graphene $\left(\right.$ Area $=10 \mathrm{~mm}^{2}$ ), Aluminium ( $\mathrm{Al}$ ) was evaporated on Si to form ohmic contact using thermal evaporation technique. Raman spectra of graphene transferred on $\mathrm{SiO}_{2} / \mathrm{n}$-Si was taken using Ar-ion gas laser operating at $488 \mathrm{~nm}$ wavelength with 600 groove/mm grating under $100 \times$ microscope objective (Trivista, Princeton Instruments). After highest occupied molecular orbital (HOMO) energy levels of TPA and CAR molecules were determined by using $\mathrm{CV}$ ( $\mathrm{CH}$ Instruments model CHI660B). KPFM measurements were employed using conductive TiN tip with curvature radius of $35 \mathrm{~nm}$ (NT-MDT). Finally, we recorded current-voltage characteristics of graphene/n-Si and graphene/SAMs/n-Si under dark condition using Keithley 2420 source meter. 


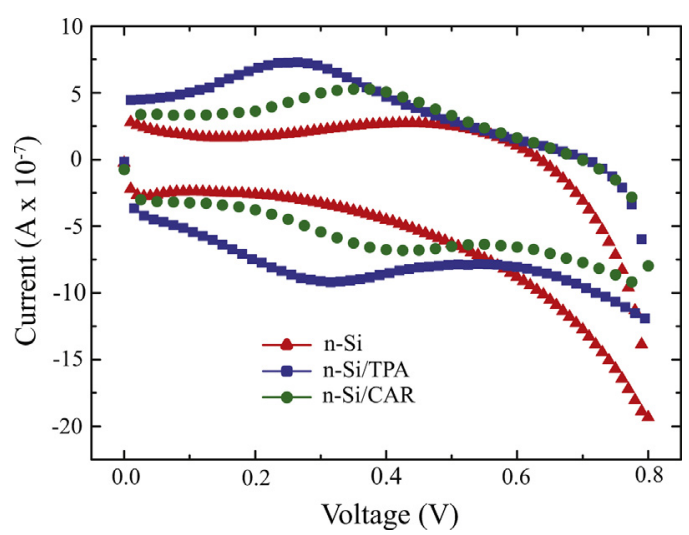

Fig. 5. Cyclic voltammograms of bare n-Si and SAMs modified n-Si with TPA and CAR molecules.

\subsection{Computational methodology}

Structural optimizations and electronic properties of TPA and CAR molecules, bilayer graphene, and Si substrate were calculated by performing ab initio density functional theory (DFT) based calculations. The Vienna ab initio simulation package, VASP [37-39] was used and the Perdew-Burke-Ernzerhof (PBE) form of the generalized gradient approximation (GGA) [40] was adopted to describe electron exchange and correlation. The vdW type weak forces are important for both SAMs systems and layered materials, hence the vdW interaction was considered in all calculations by using the DFT-D2 method of Grimme [41,42].

The kinetic energy cut-off of the plane-wave basis set was $500 \mathrm{eV}$ in all calculations. The optimization of atomic positions was performed by minimizing the total energy hence the energy difference between sequential steps was taken $10^{-5} \mathrm{eV}$ as a convergence criterion in the structural relaxation. In addition, Gaussian smearing factor of $0.01 \mathrm{eV}$ was used for non-self-consistent calculations and the pressures on the unit cell were decreased to a value less then $1.0 \mathrm{kB}$ in all three directions. Recent works of our group have shown that the DFT is powerful and reliable method to investigate the structural, electronic, and magnetic properties of SAM systems $[32,33]$.

\section{Results and discussion}

\subsection{TPA and CAR molecules}

For structural and electronic properties of diode systems consisting of TPA and CAR molecules and bilayer graphene we performed DFT based calculations. At first the single TPA and CAR molecules were investigated. Both molecules are structurally similar in which the head group of both molecules is carboxylic acid and the head-group and the spacers are linked by the terphenyl. However, the TPA and CAR have diphenylamino and carbazole as a spacer, respectively. Although the molecules have similar structures, the electronic properties are different.

As shown in Fig. 1, the calculated energy difference between the HOMO and LUMO states for the TPA and CAR molecules are 2.17 and $2.33 \mathrm{eV}$ within GGA, respectively. The level of HOMO and LUMO states are also shown in Fig. 1. For both molecules the HOMO state charge densities are localized on the spacer part, on the other hand, the LUMO state charge densities are appeared on head-group. In addition, the work functions are calculated to be 4.58 and $4.98 \mathrm{eV}$ for the TPA and CAR molecules, respectively. Such a large difference between similar molecules can be correlated with the deeper Fermi level of CAR molecule because of having strong $\mathrm{C}-\mathrm{C}$ bonds instead of $\mathrm{C}-\mathrm{H}$ bonds at carbazole part.

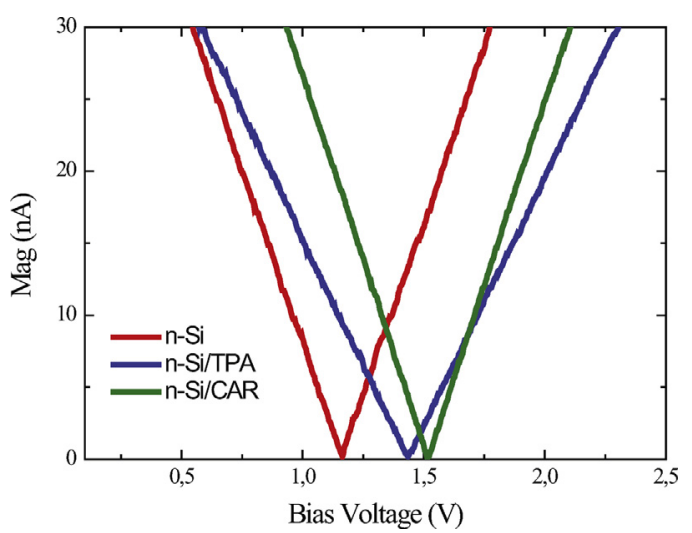

Fig. 6. Surface potential differences of bare n-Si and modified n-Si with TPA and CAR SAMs.

Table 1

$I_{\mathrm{D} / \mathrm{G}}, I_{2 \mathrm{D} / \mathrm{G}}$ and FWHM values of bilayer graphene on selected points.

\begin{tabular}{llll}
\hline & $I_{\mathrm{D} / \mathrm{G}}$ & $I_{2 \mathrm{D} / \mathrm{G}}$ & $\mathrm{FWHM} \mathrm{cm}^{-1}$ \\
\hline 1 & 0.07 & 0.99 & 40 \\
2 & 0.11 & 1.11 & 45 \\
3 & 0.13 & 1.02 & 45 \\
4 & 0.08 & 1.01 & 41 \\
\hline
\end{tabular}

In addition, considering the experimental synthesis procedure one may expect formation of defective and misoriented domains of TPA and CAR SAMs and therefore the slight differences between the experiment and DFT results in the workfunction are acceptable.

\subsection{Surface characterization}

Raman spectra indicate typical appearance of transferred graphene on $\mathrm{SiO}_{2} / \mathrm{Si}$ including D $\left(1367 \mathrm{~cm}^{-1}\right), \mathrm{G}\left(1589 \mathrm{~cm}^{-1}\right)$ and $2 \mathrm{D}$ bands $\left(2711 \mathrm{~cm}^{-1}\right)$, respectively (see Fig. 2 ). The $\mathrm{G}$ band is the first Raman peak related to $\mathrm{C}-\mathrm{C}$ stretching of $s p^{2}$ carbon. The 2D band is the second order graphene peak and the $\mathrm{D}$ band provides information about the $s p^{3}$ bonds revealing amount of disorder in graphene. Intensity ratio of $2 D / G$ and $D / G$ have been utilized to determine the number of graphene layers and defect content of graphene (see Table 1). Both intensity ratios and full width half maximum (FWHM) of 2D peak confirm the existence of BLG grown on $\mathrm{SiO}_{2} / \mathrm{n}-\mathrm{Si}[43,44]$.

Sheet resistance measurement of transferred graphene on $\mathrm{SiO}_{2} / \mathrm{Si}$ was performed using van der Pauws method to investigate quality of the graphene. In this method, while the current was measured on two isolated contacts, the voltage is determined on opposing two contacts. Eight measurements were performed around sample and the average sheet resistance of graphene was found to be $298 \Omega /$ sq. This value is in good agreement for CVD grown bilayer graphene as reported in literature $[45,46]$.

AFM image and height profile analysis of graphene film transferred on $\mathrm{SiO}_{2} / \mathrm{Si}$ are shown in Fig. 3. Some wrinkle was observed in AFM image owing to the difference thermal expansion coefficient between $\mathrm{Cu}$ surface and graphene film during the cooling process. In addition to this, thickness of bilayer graphene film was measured as $1.2 \mathrm{~nm}$ which is comparable value as reported in literature [47].

Fig. 4 demonstrates AFM images of TPA and CAR SAMs on nSi substrates. A scratch lithography in contact mode operations were performed on the TPA and CAR SAM on n-Si surfaces without scratch the substrates. TPA molecules formed uniformly on the surface. However, coverage of the CAR molecules is different as compared to TPA molecules. There are some agglomerations of CAR molecules on the surface. Thicknesses of TPA and CAR molecules 


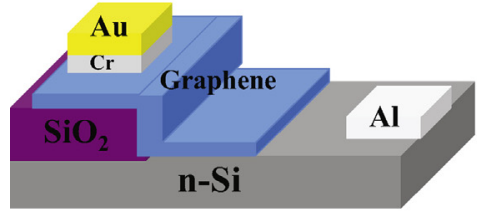

n-Si/GRP

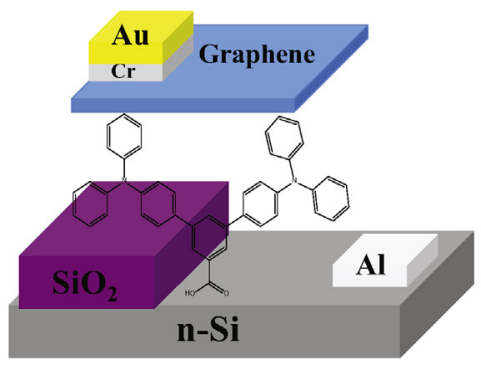

n-Si/TPA/GRP

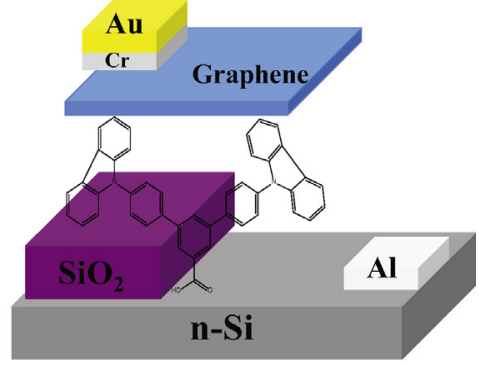

n-Si/CAR/GRP

Fig. 7. Device structure of BLG/n-Si, BLG/TPA/n-Si and BLG/CAR/n-Si.
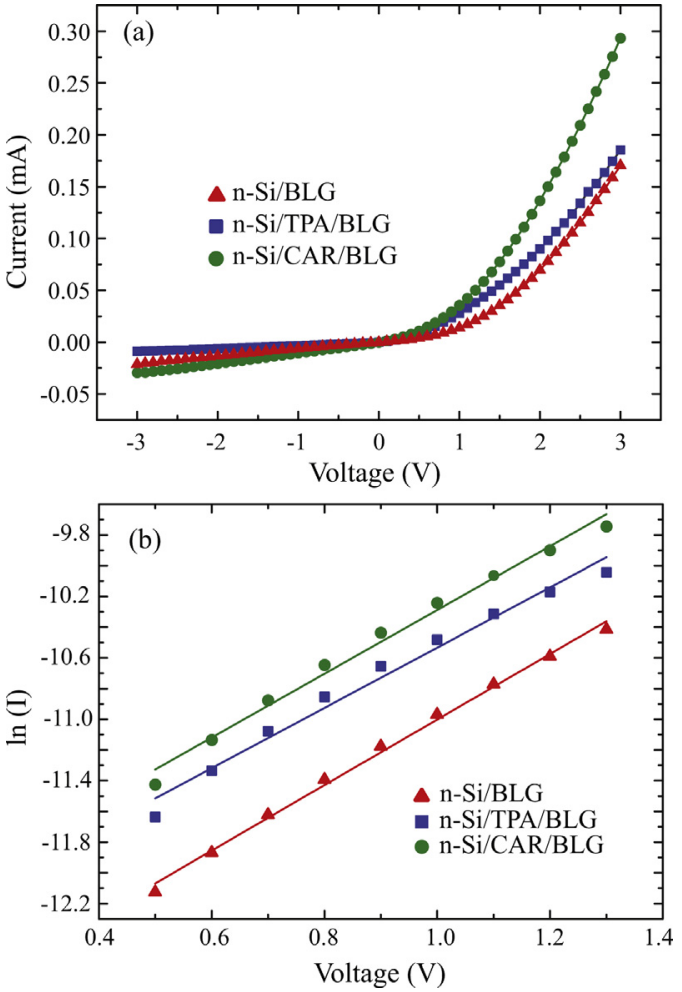

Fig. 8. (a) $I-V$ characteristic and (b) $\ln (I)-V$ plot of bare and SAMs modified Schottky devices.

were obtained from height profile as shown in Fig. 3a and b and measured as 0.8 and $1.0 \mathrm{~nm}$, respectively.

Fig. 5 indicates electrochemical property of bare TPA and CAR molecules on $\mathrm{n}$-Si surface was investigated by CV measurement in anodic region. Reversible oxidation peaks are obtained from CV voltammogram as 0.31 and $0.40 \mathrm{~V}$ for TPA and CAR SAMs. The variation in potential compare to bare n-Si reveals the carboxylic acid group anchored to n-Si surface. Additionally, HOMO levels of TPA and CAR molecules are determined from the onset of oxidation potential from the CV data using the below relation [48],

$E_{\text {НОМО }}=-e\left(E_{\text {OX }}-E_{\mathrm{Fer}}+4.8\right)$

where $E_{O X}$ is the oxidation peak and $E_{F e r}$ is the ferrocene energy which is $0.4 \mathrm{~V}$. The HOMO level values of TPA and CAR molecules were obtained to be 4.71 and $4.8 \mathrm{eV}$, respectively. The trend in the HOMO level of TPA and CAR molecules is consistent with DFT calculations.

KPFM measurements were performed on bare n-Si and SAMs modified n-Si to prove modification of TPA and CAR SAMs. Fig. 6a shows cantilever oscillation amplitude (Mag) vs bias voltage. When
Table 2

Major Schottky diodes parameters; the ideality factor, $n$; the series resistance, $R_{s}$; the Turn-on voltage, $V_{T}$; and the Schottky barrier height, $\phi_{S B H}$.

\begin{tabular}{lllll}
\hline & $n$ & $R_{S}(\mathrm{k} \Omega)$ & $V_{T}(\mathrm{~V})$ & $\phi_{\text {sbh }}(\mathrm{eV})$ \\
\hline n-Si/BLG & 2.13 & 5.96 & 0.7 & 0.54 \\
n-Si/TPA/BLG & 1.96 & 5.54 & 0.4 & 0.46 \\
n-Si/CAR/BLG & 2.07 & 4.32 & 0.3 & 0.32
\end{tabular}

Mag signal is close to zero due to feedback in the SPM system, interaction force between tip and samples becomes zero. At this zero interaction-amplitude, applied dc voltage to the tip equals to the measured SP of the samples. The SP of bare $n-S i$ and modified n-Si with TPA and CAR SAMs are found as $1.16,1.43$ and $1.51 \mathrm{~V}$, respectively. The variation in SP values indicate surface modification of n-Si surface with TPA and CAR SAMs. In addition, the difference of SP values between TPA and CAR SAMs arises from the excess carbazol groups in CAR molecular structure leading to enhance electron density of n-Si surface with respect to the TPA SAMs.

Device structure of bare and SAMs modified diodes and bonding mechanism between carboxylic acid based SAMs and n-Si with native oxide are schematically illustrated in Fig. 7.

\subsection{Current-voltage behaviour of Schottky diodes}

Driving current capability of Schottky diodes is superior to typical p-n junctions. Therefore, high current, low voltage and rectifying behaviour are desired device properties for the Schottky diode applications. The $I-V$ characteristics of BLG/n-Si and BLG/SAMs(TPA and CAR)/n-Si diodes are shown in Fig. 8a. Three devices exhibit rectification characteristic verifying Schottky junction formation at the BLG/n-Si interface. Additionally, reducing impurities and improving junction between BLG and $\mathrm{n}$-Si interface due to the presence of SAMs, result with the lower turn-on voltage $\left(V_{T}\right)$ with respect to the bare device (see Table 2). This implies that SAMs based diodes provide relatively less power wasted in comparison to the bare diode. Moreover, Schottky diode with TPA-SAMs device decreases reverse leakage whereas CAR based device exhibits high reverse leakage current. This might be attributed to agglomerations of CAR molecules on the surface as shown in AFM morphology (see Fig. 4b).

The $I-V$ characteristic of Schottky diode can be described by thermionic emission and the $R_{S}$ should be included due to the resistance of semiconductor, metal and contact with the relation [49]

$I=A A^{*} T^{2} \exp \left(-\frac{q \phi_{b}}{k T}\right)\left[\exp \left(\frac{q\left(V-I R_{s}\right)}{n k T}\right)-1\right]$

where $A$ is the effective contact area, $A^{*}$ is the Richardson constant $\left(\sim 112 \mathrm{~A} \mathrm{~cm}^{-2} \mathrm{~K}^{-2}\right.$ for $\left.\mathrm{n}-\mathrm{Si}\right), T$ is the absolute temperature, $\phi_{b}$ is the barrier height, $k$ is the Boltzmann constant, $q$ is the electronic charge and $n$ is the ideality factor. 

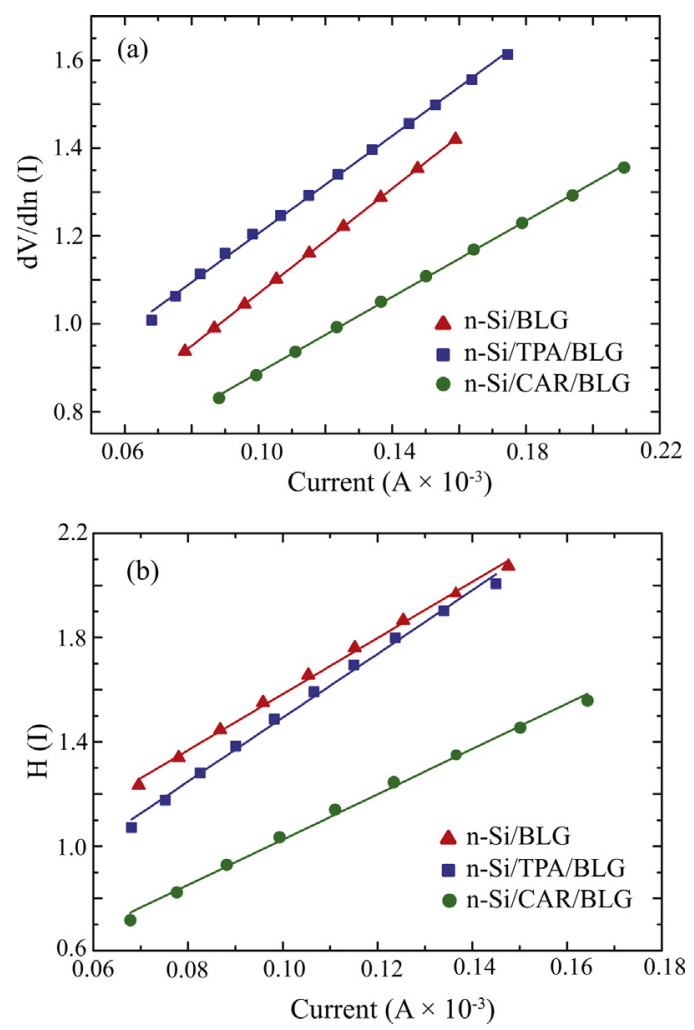

Fig. 9. (a) $d V / d \ln (I)$ vs $I$ and (b) $H(I)$ vs $I$ plots of bare and SAMs modified Schottky devices.

If Eq. (2). is rearranged,

$V=n \frac{k T}{q} \ln \left(\frac{I}{A A^{*} T^{2}}\right)+I R_{s}+n \phi_{b}$

When Eq. (3) is differentiated with respect to $I$,

$\frac{d V}{d \ln (I)}=n \frac{k T}{q}+I R_{S}$

Using Eq. (2), $n$ is determined from the slope of the linear region of $\ln (I)-V$ curve as indicated in Fig. 8b. The ideality factors of bare Schottky diode and SAMs modified Schottky diodes with TPA and CAR are calculated to be $2.13,1.96$ and 2.07 , respectively (see Table 2). The variation of ideality factors in such devices is attributed to presence of SAMs between graphene and $\mathrm{n}-\mathrm{Si}$ leading to additional interface states at the graphene-Si junction. Further, depending on difference in device fabrication, quality of graphene-Si heterojunction and data derivation in the fitting procedure, the ideality factor can be varied $[10,11,13,15,50]$.

The $R_{S}$ can be defined as the combination of contact resistance between graphene and Si, resistivity of graphene and Si and resistance of connecting wires [13]. Values of $R_{S}$ of bare and SAMs modified devices are determined from the slope of the $d V / d \ln (I)$ vs $I$ plot as shown in Fig. 9a. The calculated $R_{S}$ values, listed in Table 2, are about 5.96, 5.54 and $4.32 \mathrm{k} \Omega$ for BLG/n-Si, BLG/TPA/n-Si and $\mathrm{BLG} / \mathrm{CAR} / \mathrm{n}-\mathrm{Si}$ diodes, respectively. $R_{S}$ value of graphene/n-Si diode is comparable as reported for similar work [7]. Additionally, it is clear that $R_{S}$ of SAMs modified diodes with TPA and CAR are decreased as compare to bare device owing to improving BLG/n-Si interface. Furthermore, $R_{S}$ difference between TPA and CAR could be explained by the barrier height difference of BLG/TPA and BLG/CAR. The barrier height difference of BLG-CAR is lower than that of BLG/TPA, resulting in facilitating charge injection and lowering series resistance.
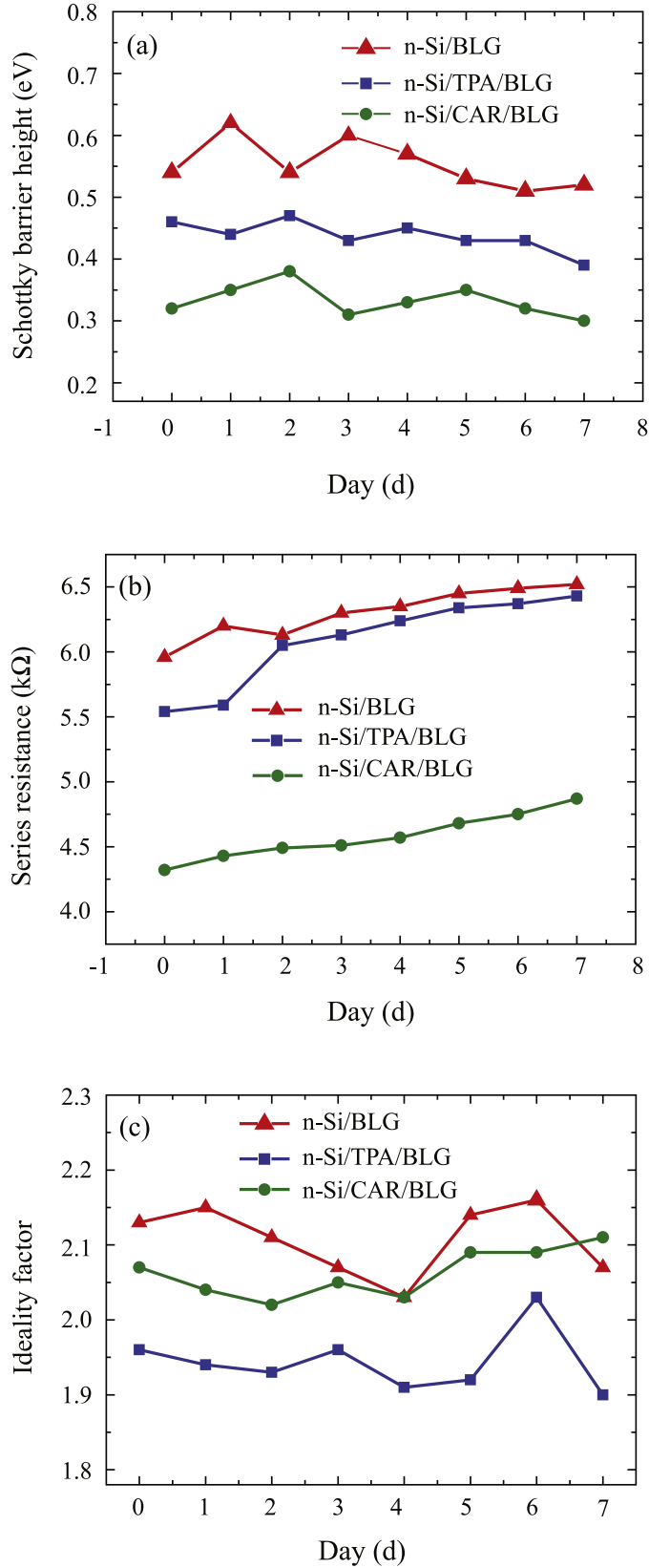

Fig. 10. Values of Schottky barrier height, series resistance and ideality factor as a function of air-exposure times up to 7 days.

In order to determine $\mathrm{SBH}$, function $H(I)$ can be defined as

$H(I)=V-n \frac{k T}{q} \ln \left(\frac{I}{A A^{*} T^{2}}\right)=I R_{S}+n \phi_{b}$

Using Eq. (5), SBH can be obtained from the intercept of the linear region of $H(I)$ vs $I$ plot as shown in Fig. 9b. The estimated SBH of $\mathrm{BLG} / \mathrm{n}-\mathrm{Si}$ diode is about $0.54 \mathrm{eV}$ which is comparable value for CVD grown graphene as reported in the literature [15]. The SBH of SAMs modified devices with TPA and CAR are calculated as 0.46 and $0.32 \mathrm{eV}$, respectively. The variation in SBH values can be explained by the different energy level of TPA and CAR SAMs. The energy barrier difference between CAR and BLG is less than the between TPA and BLG.

Fig. 10 shows values of Schottky barrier height, series resistance and ideality factor as a function of air-exposure times up to 7 days. For all devices, although the values of Schottky barrier heights and ideality factors are quite stable and they are not altered 


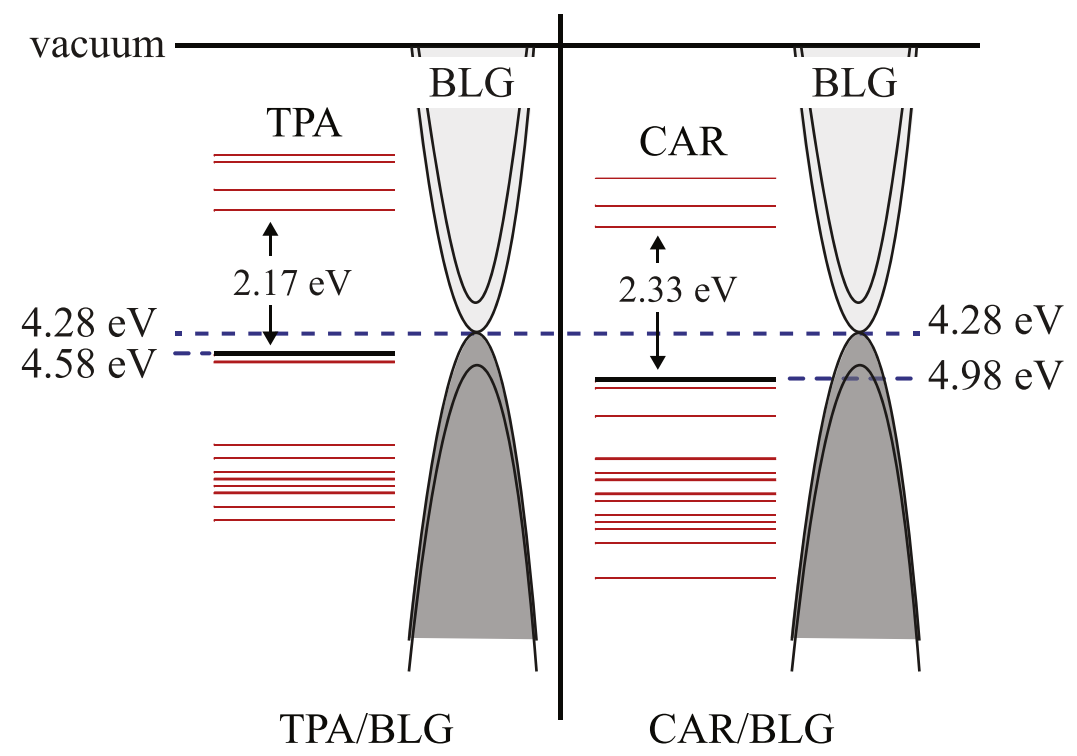

Fig. 11. The band alignments of the Si, graphene, TPA and CAR molecules with respect to their work function values.

significantly. Series resistances increase gradually with increasing air exposure times due to adsorbed molecules $\left(\mathrm{H}_{2} \mathrm{O}\right.$ and $\left.\mathrm{O}_{2}\right)$ on the graphene which give rise to degradation devices, resulting high series resistance at the interface.

As shown in Fig. 11, the CAR molecule possesses lower Schottky barrier $(1.63 \mathrm{eV})$ than the TPA molecule $(1.87 \mathrm{eV})$ with the difference of $240 \mathrm{meV}$. It is necessary to note that effects such as defective surfaces, misaligned molecules, and the PR residues lead to differences between calculated and measured barrier values. When we consider the similar synthesis procedure, such effects are also similar cumulatively for the both TPA and CAR molecules. To sum up theoretical calculations is in good agreement with the experimentally observed Schottky device characteristics.

\section{Conclusions}

In this work, we investigated electrical characteristics of bare and SAMs modified CVD grown BLG/n-Si Schottky diodes. Our results reveal that: (i) novel TPA and CAR SAMs can be used to improve graphene-Si interface, (ii) CV and KPFM confirm surface modification of $n-S i$ surface with TPA and CAR molecules, (iii) Raman spectroscopy verifies high quality $\mathrm{BLG}$ on $\mathrm{SiO}_{2} / \mathrm{Si}$, (iv) the $I-V$ characteristics of bare and SAMs modified diodes show rectification behaviour confirming Schottky formation at the interface, (v) SAMs modified devices exhibit better diode characteristic in terms of turn-on voltage, $R_{S}$ and $\mathrm{SBH}$, (vi) the variation in device performance between TPA and CAR based diodes is related to lower energy barrier difference of CAR/BLG system as compared to that of TPA/BLG, resulting in reduction of turn-on voltage, SBH and $R_{s}$, (vii) theoretical calculations also verify that better Schottky device characteristics of SAMs of CAR/BLG are directly related to the order of HOMO-LUMO levels in energy space that leads to a lower energy barrier than TPA/BLG interface. Apparently, our results show that robust characteristics, structural stability and lower Schottky barriers of SAM/graphene-based interfaces hold promise for novel ultra-thin optoelectronic devices.

\section{Acknowledgements}

This work was supported by TUBITAK (The Scientific and Technical Research Council of Turkey) with project number $112 \mathrm{T946.}$ We also thank AQuReC (Applied Quantum Research Center) for
Raman measurements. Computational resources were provided by TUBITAK ULAKBIM, High Performance and Grid Computing Center (TR-Grid e-Infrastructure). H.S. acknowledges support from Bilim Akademisi - The Science Academy, Turkey under the BAGEP program.

\section{References}

[1] K.S. Novoselov, A.K. Geim, S.V. Morozov, D. Jiang, M.I. Katsnelson, I.V. Grigorieva, S.V. Dubonos, A.A. Firsov, Nature (London) 438 (2005) 197

[2] A.K. Geim, K.S. Novoselov, Nat. Mater. 6 (2007) 183.

[3] K.I. Bolotin, K.J. Sikes, Z. Jianga, M. Klimac, G. Fudenberga, J. Honec, P. Kima, H.L. Stormera, Solid State Commun. 146 (2008) 351.

[4] H. Yang, J. Heo, S. Park, H.J. Song, D.H. Seo, K.-E. Byun, P. Kim, I. Yoo, H.-J Chung, K. Kim, Science 336 (2012) 1140.

[5] B.X. Li, H. Zhu, K. Wang, A. Cao, J. Wei, C. Li, Y. Jia, Z. Li, X. Li, D. Wu, Adv. Mater 22 (2010) 2743.

[6] X. Wang, Z. Cheng, K. Xu, H.K. Tsang, J.-B. Xu, Nat. Photon. 7 (2013) 888.

[7] H.-Y. Kim, K. Lee, N. McEvoy, C. Yim, G.S. Duesberg, Nano Lett. 13 (2013) 2182

[8] D. Tomer, S. Rajput, L.J. Hudy, C.H. Li, L. Li, Nanotechnology 26 (2015) 215702.

[9] S. Shivaraman, L.H. Herman, F. Rana, J. Park, M.G. Spencer, Appl. Phys. Lett. $100(2012) 183112$

[10] S. Tongay, M. Lemaitre, T. Schumann, K. Berke, B.R. Appleton, B. Gila, A.F. Hebard, Appl. Phys. Lett. 99 (2011) 102102.

[11] G. Kalita, R. Hirano, M.E. Ayhan, M. Tanemura, J. Phys. D: Appl. Phys. 46 (2013) 455103.

[12] Y.-J. Lin, J.-H. Lin, Appl. Surf. Sci. 311 (2014) 224.

[13] D.-J. Kim, G.-S. Kim, N.-W. Park, W.-Y. Lee, Y. Sim, K.-S. Kim, M.-J. Seong, J.-H. Koh, C.-H. Hong, S.-K. Lee, J. Alloys Compd. 612 (2014) 265.

[14] Y. An, A. Behnam, E. Pop, A. Ural, Appl. Phys. Lett. 102 (2013) 013110.

[15] D. Sinha, J.U. Lee, Nano Lett. 14 (2014) 4660.

[16] M. Mohammed, Z. Li, J. Cui, T.-P. Chen, Nanosc. Res. Lett. 7 (2014) 302.

[17] S. Tongay, M. Lemaitre, X. Miao, B. Gila, B.R. Appleton, A.F. Hebard, Phys. Rev. X 2 (2012) 011002.

[18] D. Tomer, S. Rajput, L.J. Hudy, C.H. Li, L. Li, Appl. Phys. Lett. 106 (2015) 173510

[19] A. Ulman, Chem. Rev. 96 (1996) 1533.

[20] J.C. Love, L.A. Estroff, J.K. Kriebel, R.G. Nuzzo, G.M. Whitesides, Chem. Rev. 105 (2005) 1103

[21] M. Lafkioti, B. Krauss, T. Lohmann, U. Zschieschang, H. Klauk, K.V. Klitzing, J.H Smet, Nano Lett. 10 (2010) 1149.

[22] X. Wang, J.-B. Xu, C. Wang, J. Du, W. Xie, Adv. Mater. 23 (2011) 2464.

[23] S. Wang, S. Suzuki, K. Furukawa, C.M. Orofeo, M. Takamura, H. Hibino, Appl. Phys. Lett. 103 (2013) 253116

[24] H. Lv, H. Wu, K. Xiao, W. Zhu, H. Xu, Z. Zhang, H. Qian, Appl. Phys. Lett. 102 (2013) 183107.

[25] N. Cernetic, D.O. Hutchins, H. Ma, A.K.-Y. Jen, Appl. Phys. Lett. 106 (2015) 021603.

[26] B. Lee, Y. Chen, F. Duerr, D. Mastrogiovanni, E. Garfunkel, E.Y. Andrei, V. Podzorov, Nano Lett. 10 (2010) 2427

[27] Na. Cernetic, S. Wu, J.A. Davies, B.W. Krueger, D.O. Hutchins, X. Xu, H. Ma, A.K.-Y. Jen, Adv. Mater. 24 (2014) 3464

[28] K. Yokota, K. Takai, T. Enoki, Nano Lett. 11 (2011) 3669. 
[29] W.H. Lee, J. Park, Y. Kim, K.S. Kim, B.H. Hong, K. Cho, Adv. Mater. 23 (2011) 3460.

[30] Z. Yan, Z. Sun, W. Lu, J. Yao, Y. Zhu, J.M. Tour, ACS Nano 5 (2011) 1535.

[31] S. Lenfant, C. Krzeminski, C. Delrue, G. Allan, D. Vuillaume, Nano Lett. 3 (2003) 741.

[32] A. Tomak, C. Bacaksiz, G. Mendirek, H. Sahin, D. Hur, K. Görgün, R.T. Senger, Ö Birer, F.M. Peeters, H.M. Zareie, Nanotechnology 27 (2016) 33.

[33] D. Kiymaz, M. Yagmurcukardes, A. Tomak, H. Sahin, R.T. Senger, F.M. Peeters, H.M. Zareie, C. Zafer, Nanotechnology 27 (2016) 45.

[34] N. Yagmurcukardes, H. Aydin, M. Can, A. Yanlmaz, O. Mermer, S. Okur, Y. Selamet, ECS J. Solid State Sci. Technol. 5 (2016) M69.

[35] T. Yu, F. Wang, Y. Xu, L. Ma, X. Pi, D. Yang, Adv. Mater. 28 (2016) 4912.

[36] Y. Jeong, J. Wook Han, N. Kim, Y. Lee, C. Lee, M. Hara, J. Noh, Bull. Korean Chem. Soc. 28 (2007) 2445.

[37] G. Kresse, J. Hafner, Phys. Rev. B 47 (1993) 558

[38] G. Kresse, J. Furthmller, Phys. Rev. B 54 (1996) 11169.

[39] G. Kresse, D. Joubert, Phys. Rev. B 59 (1999) 1758.

[40] J.P. Perdew, K. Burke, M. Ernzerhof, Phys. Rev. Lett. 77 (1996) 3865.
[41] S.J. Grimme, Comput. Chem. 27 (2006) 1787.

[42] T. Bucko, J. Hafner, S. Lebegue, J.G. Angyan, J. Phys. Chem. A 114 (2010) 11814.

[43] A.C. Ferrari, J.C. Meyer, V. Scardaci, C. Casiraghi, M. Lazzeri, F. Mauri, S.

Piscanec, D. Jiang, K.S. Novoselov, S. Roth, A.K. Geim, Phys. Rev. Lett. 97 (2006) 187401.

[44] W. Liu, S. Kraemer, D. Sarkar, H. Li, P.M. Ajayan, K. Banerjee, Chem. Mater. 26 (2013) 907.

[45] S. Chen, W. Cai, R.D. Piner, J.W. Suk, Y. Wu, Y. Ren, J. Kang, R.S. Ruoff, Nano Lett. 11 (2011) 3519.

[46] M. Madito, A. Bello, J. Dangbegnon, C. Oliphant, W. Jordaan, D. Momodu, T. Masikhwa, F. Barzegar, M. Fabiane, N. Manyala, J. Appl. Phys. 119 (2016) 015306.

[47] A. Reina, X. Jia, J. Ho, D. Nezich, H. Son, V. Bulovic, M.S. Dresselhaus, J. Kong, Nano Lett. 9 (2008) 30

[48] L. Leonat, G. Sbrcea, I.V. Brnzoi, U.P.B. Sci. Bull. Ser. B 75 (2013) 111.

[49] A. Mekki, R.O. Ocaya, A. Dere, A.A.A. -Ghamdi, K. Harrabi, F. Yakuphanoglu, Synth. Metals 213 (2016) 47.

[50] N.-W. Park, W.-Y. Lee, S.-K. Lee, J. Korean Phys. Soc. 66 (2015) 22. 